\title{
Mesoscopic Thermodynamics in the Presence of Flow
}

\author{
I. Santamaría-Holek ${ }^{1}$, R. Lugo-Frías ${ }^{1}$, R. F. Rodríguez ${ }^{2}$ and A. Gadomski ${ }^{3}$ \\ ${ }^{1}$ UMJ-Facultad de Ciencias, Universidad Nacional Autónoma de México \\ ${ }^{2}$ Instituto de Física, Universidad Nacional Autónoma de México \\ ${ }^{3}$ Institute of Mathematics \& Physics, University of Technology \& Life Sciences \\ ${ }^{1,2}$ México \\ ${ }^{3}$ Poland
}

\section{Introduction}

Thermodynamics is a powerful theory that has been succesfully applied to describe the properties and behavior of macroscopic systems under a very wide range of physical conditions (Callen, 1985). Thermal and caloric information is obtained by performing experiments that agree with the constraints imposed (Callen, 1985; Kondepudi \& Prigogine, 1999; Ragone, 1995).

However, a considerable number of experimental, practical problems and systems of interest are often found in nonequilibrium or metastable states in which static thermodynamic relations are only valid locally, and frequently are insufficient to describe complicated time dependent situations (Demirel, 2007). Macroscopic systems in flow conditions are good examples of this peculiar behavior through non-Newtonian rheological effects, phase transitions and generalized statistics of turbulent motion. In fact, although there is a large amount of theoretical work proposing generalizations of the thermodynamic theory to nonequilibrium or quasiequilibrium situations (Beck \& Cohen, 2003; Beris \& Edwards, 1994; C. Beck \& Swinney, 2005; de Groot \& Mazur, 1984; Demirel, 2007; Kondepudi \& Prigogine, 1999; Onuki, 2004; Rodríguez \& Santamaría Holek, 2007), some of them very succesful (de Groot \& Mazur, 1984; Demirel, 2007; Kondepudi \& Prigogine, 1999), they are restricted due to the assumption and validity of local thermodynamic equilibrium. Recently, some interesting generalizations of thermodynamics to describe macroscopic systems in the presence of flow have been systematically developed in Refs. (Beris \& Edwards, 1994; Onuki, 2004). Nevertheless, there are still a good deal of open questions, for instance those concerning the validity of the usual relations for the thermal and caloric equations of state, arising further research on this subject.

Related to these general questions, there are several particular manifestations of the effects of flow on the thermodynamic behavior of systems. For example, diffusion of suspended particles in a heat bath in equilibrium, may strongly differ from that when the particles diffuse in a heat bath under the presence of shear flow. These effects have been analyzed along the years in studies ranging from experiments (Breedveld, 1998; Guasto \& Gollub, 2007; Pine, 2005; Taylor \& Friedman, 1996) and computer simulations (Sarman, 1992) to kinetic 
theory of gases (Rodríguez, 1983), projector operator techniques (Shea \& Oppenheim, 1998), Langevin and Fokker-Planck dynamics (Drossinos \& Reeks, 2005; Mauri \& Leporini, 2006; Ryskin, 1988; Subramanian \& Brady, 2004) and mesoscopic thermodynamics (Gadomski, 2008; Santamaría Holek, 2005; 2009; 2001).

The key point in these studies is that the presence of flow introduces non-thermal diffusion effects that can be related to the hydrodynamic viscosity and to hydrodynamic interactions between particles in the case of concentrated suspensions. These effects may modify the fluctuation-dissipation relations and, consequently, the corresponding expressions for the thermal energy and other state variables like pressure or chemical potential. This may lead to what is known as nonequilibrium state equations (Onuki, 2004).

In this chapter, we present a discussion of the general context involving thermodynamics in the presence of flows, emphasizing how the flow may modify the state equations as well as the transport coefficients. A selected presentation of some experimental results in which the effect of the flow clearly modifies the thermodynamics of the system is given and discussed in view of theoretical models attempting to describe them, mainly those coming from mesoscopic thermodynamics. How non-Newtonian effects (Santamaría Holek, 2005) arise from nonequilibrium state equations, how the transition to irreversibility in suspensions of non-Brownian particles ocurrs (Breedveld, 1998; Guasto \& Gollub, 2007; Pine, 2005; Santamaría Holek, 2009; Taylor \& Friedman, 1996) and how the nucleation in the presence of shearing flows is promoted or supressed (A. Penkova, 2006; Blaak, 2004), are the three main topics that we will analyze in more detail.

The original contribution of this chapter bears on the discussion of two recent studies on the problem of nucleation in the presence of flows (Blaak, 2004; Reguera \& Rubi, 2003b) and the formulation of a new model that allows to explain experimental results (A. Penkova, 2006). These models are excellent illustrations of how the effects of flow affect the equations of state of fluid systems. Particularly, we will show how control volume thermodynamics in the presence of flow, a well established analysis of classical thermodynamics (Ragone, 1995), can be used to formulate a qualitative theoretical explanation of the experimental observations. This chapter is organized as follows. Section 2 is devoted to analyze the effects of flow on diffusion from different points of view ranging from kinetic theory of gases, Langevin equation approach and mesoscopic nonequilibrium thermodynamics. In Section 3 we analyze how flow modifies the constitutive relations of materials via viscoelastic and non-Newtonian effects. Thereafter, the existence of nonquilibrium equations of state is discussed in Section 4 and then used to describe nucleation in the presence of flow in Section 5. Finally, we present our conclusions in Section 6.

\section{Effects of flow on diffusion}

The effects of flow on the diffusion of particles suspended in a simple liquid is an important problem which was analyzed many times along the last 30 years. Several approaches have been followed in order to understand and quantify the effects of the presence of a velocity gradient on the diffusion coefficient $D$ of the particles. Usually, these are restricted to the case of shear flow because this case is more manageable from the mathematical point of view, and because there are several experimental systems that allow the evaluation and validity of the corresponding results. The approaches followed vary from kinetic theory and projector operator techniques, to Langevin and Fokker-Planck equations. Here, we summarize some recent contributions to this subject and their results. 


\subsection{Kinetic theory approach.}

From a rigorous point of view, the problem of diffusion in the presence of flow should be analyzed by means of the kinetic theory gases. This has been done in a series of articles (Refs. (Ferziger \& Kaper, 1972; Marchetti \&Dufty, 1983; McLennan, 1989; Rodríguez, 1983)) and lead to a better understanding of the phenomenology presented by these systems. The rigorous derivations coming from kinetic theory serve to indicate and evaluate the range of validity of those coming from different approaches, some of them will be discussed later.

Essentially, the problem consists in the coupling of the dynamics of two subsystems, the host fluid and an ensemble of tagged particles that, in the case of massive and non-concentrated particles, constitute an ideal Brownian gas. Each susbsystem can be described by means of their corresponding phase space densities that evolve in time following a set of coupled Boltzmann equations.

In first approximation (Marchetti \&Dufty, 1983), the system considered is a tagged particle in a low density gas of Maxwell molecules interacting through a $r^{-5}$ force law. The fluid is assumed to be in a state of uniform shear flow with a large shear rate and the tagged particle is considered to be mechanically equivalent but with a larger mass than the fluid particles. The fluctuations of the tagged particle in the nonequilibrium gas can be described kinetically using the nonlinear Boltzmann and the Boltzmann-Lorentz equations.

$$
\begin{aligned}
& \left(\frac{\partial}{\partial t}+\mathbf{v} \cdot \nabla\right) f=J[f, f] \\
& \left(\frac{\partial}{\partial t}+\mathbf{v} \cdot \nabla\right) h=J[f, h]
\end{aligned}
$$

where $f(\mathbf{x}, t)$ and $h(\mathbf{x}, t)$ are the phase space densities for the fluid and tagged particle, and $\mathbf{x}$ denotes the position and velocity of the later. $J[f, f]$ is the usual bilinear Boltzmann operator (Ferziger \& Kaper, 1972; McLennan, 1989). The previous pair of equations is completed considering the evolution equation for the fluctuations of the tagged particle $C_{1,2}$

$$
\left(\frac{\partial}{\partial t}+\mathbf{v}_{1} \cdot \nabla_{1}\right) C_{1,2}=J\left[f, C_{1,2}\right]
$$

where

$$
C_{1,2}\left(\mathbf{x}_{1}, t+\tau ; \mathbf{x}_{2}, \tau\right)=\left\langle\delta\left[\mathbf{x}_{1}-\mathbf{x}_{T}(t+\tau)\right] \cdot\left\{\delta\left[\mathbf{x}_{2}-\mathbf{x}_{T}(\tau)\right]-\left\langle\delta\left[\mathbf{x}_{2}-\mathbf{x}_{T}(\tau)\right]\right\rangle\right\}\right\rangle
$$

In the special case of Maxwell molecules with uniform shear flow one can obtain an exact set of kinetic equations for the average position and velocity, as well as their fluctuations, from Eqs. (1)-(3).

This kinetic description can be extended to the case where the tagged and fluid particles are assumed to interact via the same force law that holds for fluid particles. The mass $m$ of the tagged particle is assumed to be large compared to that of a fluid particle $\left(m_{f}\right)$. Therefore, the mass ratio $\epsilon=m_{f} / m$ is a small parameter in terms of which the Boltzmann- Lorentz collision operator may be expanded. If this expansion is carried out to the leading order, $\epsilon^{1 / 2}$, the Boltzmann-Lorentz operator reduces to a differential operator yielding a kinetic Fokker Planck equation for the tagged particle distribution $F$

$$
\left(\frac{\partial}{\partial t}+\mathbf{v} \cdot \nabla\right) F=\frac{\partial}{\partial v_{i}}\left[A_{i}(\mathbf{r}, \mathbf{v}, t)+\frac{1}{2} \frac{\partial}{\partial v_{j}} D_{i j}(\mathbf{r}, t)\right] F,
$$


where the drift vector $A_{i}(\mathbf{r}, \mathbf{v}, t)$ and the diffusion tensor $D_{i j}(\mathbf{r}, t)$ are given by

$$
\begin{gathered}
A_{i}(\mathbf{r}, \mathbf{v}, t)=v_{1} \frac{n(\mathbf{r}, t)}{n_{0}}\left[\mathbf{v}-\mathbf{v}_{0}(\mathbf{r}, t)\right], \\
D_{i j}(\mathbf{r}, t)=2 \rho_{0}^{-1}\left[v_{1} p(\mathbf{r}, t) \delta_{i j}+\left(v_{1}-v_{2}\right) \mathbb{P}_{i j}^{*}(\mathbf{r}, t)\right] .
\end{gathered}
$$

Here, $v_{1}$ and $v_{2}$ are constants proportional to $\epsilon^{1 / 2}$, and $n(\mathbf{r}, t), p(\mathbf{r}, t)$ and $\mathbf{v}_{0}(\mathbf{r}, t)$ are the local density, pressure and flow velocity of the fluid. The constant $n_{0}$ is the average number density and $\rho_{0}=m n_{0}$. Finally, $\mathbb{P}_{i j}^{*}(\mathbf{r}, t)$ is the traceless part of the fluid pressure tensor and represents the irreversible part of the momentum flux proportional to $\nabla \mathbf{v}_{0}$.

Although equation (7) is valid for an arbitrary nonequilibrium state of the fluid, for a fluid in uniform shear flow $v_{0, i}(\mathbf{r})=\dot{\gamma}_{i j} r_{j}$, where $\dot{\gamma}_{i j}$ is the rate tensor, there are important simplifications. In this case the drift vector remains the same, but the diffusion tensor becomes independent of $\mathbf{r}$ and $\mathbf{v}$

$$
D_{i j}(\mathbf{r}, t)=2 v_{1}\left[k_{B} T(t) / m\right] \delta_{i j}+2\left(v_{1}-v_{2}\right) \rho_{0}^{-1} \mathbb{P}_{i j}^{*}(t) .
$$

The results obtained from a kinetic description show that the drift vector and the diffusion tensor can be exactly calculated and that they depend on the nonequilibrium state of the gas only through the low order moments of the fluid distribution function. Even more, the fluid state in this case is specified by the nonequilibrium temperature $T(t)$ and the irreversible stress tensor $\mathbb{P}_{i j}^{*}(t)$ which is proportional to the velocity gradient $\nabla \mathbf{v}_{0}$.

These results imply that the extension of equilibrium theories to nonequilibrium states is not always valid in a straightforward way. Particularly, the diffusion tensor is proportional to the components of the pressure tensor or equivalently to the velocity gradient $\nabla \mathbf{v}_{0}$, which implies that the amplitude of the noise in the dynamics of the tagged particle is not simply thermal as in equilibrium since the diffusion tensor cannot be characterized entirely by the thermodynamic temperature. In similar manner, Eq. (5) does not depend on the irreversible heat flux. This is an anomaly of the Maxwell potential, for other potentials there will be an additional contribution to the drift vector that would depend on the any temperature gradient in the fluid.

\subsection{Langevin equation approach}

Another interesting approach to the effect of flow on diffusion is the one based on Langevin equations (Drossinos \& Reeks, 2005; Swailes, 2009). Related to this approach is the interesting discussion on the existence and generality of effective nonequilibrium temperatures, which attracted much attention in recent years (Kurchan, 2005; Mauri \& Leporini, 2006; Pérez-Madrid, 2005; Pérez-Madrid \& Santamaría Holek, 2009; Popov \& R., 2007). The possibility of using thermodynamic quantitites like the temperature or the chemical potential in systems out of equilibrium is attractive because it may strongly simplify the solutions to many practical problems (Demirel, 2007).

Specifically, the existence of a nonequilibrium temperature in sheared systems lead to a considerable theoretical (Criado-Sancho, 2005; Mauri \& Leporini, 2006), numerical (Kurchan, 2005; O'Hern, 2004; Sarman, 1992) and experimental works with particular emphasis to the nonequilibrium properties of colloidal suspensions(Abou \& Gallet, 2004; Bellon, 2001). These studies are relevant to us because kinetic nonequilibrium temperatures may not follow equipartition and are usually related to the breakdown of the fluctuation-dissipation theorem, 
a central relation of Langevin descriptions (Criado-Sancho, 2005; Santamaría Holek, 2001; Subramanian \& Brady, 2004).

Suspended objects diffuse in and are convected by the fluid; their motion may eventually be influenced by the presence of external fields. In Ref. (Drossinos \& Reeks, 2005) the simultaneous diffusive and inertial motion of particles in simple shear flow is investigated to determine the effect of particle inertia on diffusive transport. First, a mesoscopic approach is used to describe the motion of a rigid spherical Brownian particle in a two dimensional simple shear flow with shear rate tensor $\dot{\gamma}_{i j},(i, j=x, y)$, with a Langevin equation in a Lagrangian reference frame. In this model the fluid velocity is along the $x$-direction, $v_{i}=\dot{\gamma}_{i j} r_{j}$, and the only nonzero element of $\dot{\gamma}_{i j}$ is $\dot{\gamma}_{x y}=\dot{\gamma}$. However, since the particle density $\left(\rho_{p}\right)$ is much larger than the fluid density, $\left(\rho_{f}\right)$, modifications to Stokes drag such as Basset history integral or Faxen's corrections, are neglected. Furthermore, only small-diameter, low-inertia particles $(a \geq 50 \mu m$ ), are considered so that the gravitational settling and the Saffman lift force may be also neglected. The particle equation of motion is

$$
\frac{d}{d t} \mathbf{u}=\beta(\underline{\dot{\gamma}} \cdot \mathbf{r}-\mathbf{u})+\mathbf{f}(t),
$$

where the random force per unit particle mass, $\mathbf{f}(t)$, is taken to be white in time,

$$
\left\langle f_{i}(t) f_{j}\left(t^{\prime}\right)\right\rangle=q \delta_{i j} \delta\left(t-t^{\prime}\right),
$$

The friction coefficient $\beta$ is the inverse particle's relaxation time, $\beta=9 \mu_{f} /\left(2 \rho_{p} a^{2}\right)$, where $\mu_{f}$ is the fluid's dynamic viscosity. Since the Langevin equations are linear, particle velocity and position may be formally solved as functionals of the random force, and in the diffusive limit $t>>\beta^{-1}$, i. e., for times much larger than the particle relaxation time, they allow for the analytical evaluation of ensemble averaged products of particle position and velocity and two-point correlation functions, in terms of the random-force strength $q$. The authors carefully justify why they use the classical (equilibrium) form of the fluctuation-dissipation theorem (FDT): in a Langevin description the time scale of the white noise is considered to be much shorter than the time scale of the imposed flow. Thus, the non-equilibrium corrections would be of the order of the ratio of the fluid molecular relaxation time to the time scale of the imposed shear and may be neglected. In this case both the time scales are clearly separated and $q$ may be determined solely from the classical form of the FDT,

$$
\frac{q}{2 \beta}=\frac{k_{B} T}{m} \text {. }
$$

In the diffusion limit it is found that the combined effects of particle inertia and shear flow modify the amplitude and the time-dependence of the particle-velocity autocorrelation functions, a result which is expressed in terms of the Stokes number, $S t=\dot{\gamma} / \beta$. The shear flow breaks macroscopic time reversibility and stationarity: the autocorrelation functions of the particle velocities are stationary and the velocity correlation along the shear is symmetric in the time difference $\tau$, but the cross correlation is non-symmetric in $\tau$ function in the streamwise direction is non-stationary. The time decay of the velocity correlation along the flow is not a pure exponential and the underlying stochastic process is not an Ornstein-Uhlenbeck process. 
Secondly, the authors give an Eulerian description of the motion of $N$ independent and identical Brownian particles in terms of the phase space density $f(\mathbf{r}, \mathbf{u} ; t)$ obeying the Fokker-Planck equation (FPE)

$$
\frac{\partial f}{\partial t}+\nabla \cdot(\mathbf{u} f)=\beta \frac{\partial}{\partial \mathbf{u}} \cdot[(\mathbf{u}-\mathbf{v}) f]+\frac{k_{B} T}{m} \beta \frac{\partial^{2} f}{\partial \mathbf{u}^{2}},
$$

where $\nabla \equiv \partial / \partial \mathbf{r}$ and the classical FDT has also been used. The solution of this equation defines a Gaussian process which is used to evaluate density-weighted ensemble averages like particle concentrations, mean particle velocities and particle-velocity covariances. In particular, by taking velocity moments of the FPE the appropriate coupled mass and momentum equation for the particle phase are obtained for the case of a general flow and a white noise random force,

$$
\begin{gathered}
\frac{d \rho}{d t}=-\rho \nabla \cdot\langle\mathbf{v}\rangle \\
\frac{d}{d t}\langle\mathbf{u}\rangle=\beta(\mathbf{v}-\langle\mathbf{u}\rangle)-\frac{1}{\rho} \nabla \cdot\left(\rho\left\langle\mathbf{u}^{\prime} \mathbf{u}^{\prime}\right\rangle\right) .
\end{gathered}
$$

Here $\mathbf{v}^{\prime}=\mathbf{v}-\langle\mathbf{v}\rangle$ is the fluctuating component of the velocity and the overbar or angular brackets denote an average over a normalized velocity probability density. Thirdly, by using the analytical solution of the FPE, in conjuction with its first two-velocity moment equations given above, a convective-diffusion equation, or generalized Smoluchowski equation (GSE), may be derived without the use of non-perturbative approximations in the diffusive limit,

$$
\frac{\partial \rho}{\partial t}+\nabla \cdot(\rho \mathbf{v})=\langle(\mathbf{u}-\mathbf{v})\rangle \mathbf{r} \cdot \nabla^{2} \rho \equiv \mathbb{D} \cdot \nabla^{2} \rho .
$$

This equation incorporates inertial effects on diffusional transport for dilute suspensions and defines the diffusion tensor, $\mathbb{D}(S t)$, dependent on both particle inertia and the shear rate through the Stokes number $S t$.

The GSE is valid in the transition regime between the diffusion limit and the inertia-dominated limit and incorporates both particle transport mechanisms. The diffusion tensor is not symmetric reflecting the symmetry-breaking effect of the imposed shear. The total diffusion coefficients measuring the particle mean square displacement are always positive and depend on the shear rate and particle inertia. However, the stream-wise diffusion coefficient becomes negative with increasing Stokes number and one of the cross coefficients is always negative.

\subsection{Mesoscopic nonequilibrium thermodynamics approach}

A different formalism in which the diffusion of a Brownian gas in a fluid under stationary and non-stationary flow has been analyzed is mesoscopic nonequilibrium thermodynamics (MNET) (Pérez-Madrid, 1994; Rubi \& Mazur, 1994; Rubi \& Pérez-Madrid, 1999). This theory uses the nonequilibrium thermodynamics rules in the phase space of the system, and allows to derive Fokker-Planck equations that are coupled with the thermodynamic forces associated to the interaction between the system and the heat bath. The effects of this coupling on system's dynamics are not obvious. This is the case of Brownian motion in the presence of flow where, as we have discussed previously, both the diffusion coefficient and the chemical potential become modified by the presence of flow (Reguera \& Rubi, 2003a;b; Santamaría Holek, 2005; 2009; 2001).

Using the principles of MNET, a thermodynamics for systems in a stationary state induced by an imposed flow was formulated based on the assumption of the existence of local equilibrium 
in the phase space of the system (Santamaría Holek, 2005). This assumption enables one to interpret the probability density and its conjugated nonequilibrium chemical potential, as mesoscopic thermodynamic variables.

\subsection{Mesoscopic nonequilibrium thermodynamics in the presence of flows}

As in the Langevin description, the dynamic description of noninteracting Brownian particles moving in a fluid in stationary flow, demands a mesoscopic treatment in terms of the probability density $f(\mathbf{r}, \mathbf{u}, t)$. The evolution in time of this quantity is governed by the continuity equation

$$
\frac{\partial f}{\partial t}+\nabla \cdot(\mathbf{u} f)=-\frac{\partial}{\partial \mathbf{u}} \cdot\left(f \mathbf{V}_{\mathbf{u}}\right),
$$

where $f \mathbf{V}_{\mathbf{u}}$ is a probability current in $\mathbf{u}$-space. The analysis of the dynamics starts from the entropy production rate $\sigma(t)$ related to the probability diffusion process. This can be done by invoking the second principle of thermodynamics that the entropy production rate should be positive definite $\sigma(t) \geq 0$. To calculate $\sigma(t)$ it is convenient to use the relative canonical entropy, or generalized Gibbs entropy postulate (de Groot \& Mazur, 1984; Pérez-Madrid, 1994; Rubi \& Mazur, 1994),

$$
\rho \Delta s(t)=-k_{B} \int f \ln \left|f / f_{l e q}\right| d \Gamma,
$$

where $k_{B}$ is Botzmann's constant, $\Delta s=s-s_{\text {leq }}$ is the difference of the specific nonequilibrium entropy $s(t)$ with respect to that characterizing the local equilibrium reference state $s_{l e q}$, and $d \boldsymbol{\Gamma}=d \mathbf{r} d \mathbf{u}$. In addition, the mass density of the system of particles is defined by

$$
\rho(\mathbf{r}, t)=m \int f(\mathbf{r}, \mathbf{u}, t) d \mathbf{u},
$$

where $m$ is the mass of a Brownian particle. Eq. (17) constitutes an irreversibility criterion assuring that the obtained equations satisfy an extremal principle in $\Gamma$-space.

The local equilibrium distribution function $f_{l e q}(\mathbf{r}, \mathbf{u})$ can be described by the local Maxwellian with respect to the non-stationary convective flow $\mathbf{v}_{0}(\mathbf{r}, t)$, that is

$$
f_{l e q}(\mathbf{r}, \mathbf{u})=e^{-\frac{m}{k_{B} T}\left[\mu_{B}+\frac{1}{2}\left(\mathbf{u}-\mathbf{v}_{0}\right)^{2}\right]},
$$

where $\mu_{B}$ is the chemical potential at equilibrium. Using these ingredients, the balance equation for the internal energy and assuming isothermal conditions, the entropy production rate of the system was obtanied in the form (Santamaría Holek, 2005; 2001)

$$
\sigma=-\frac{m}{T} \int f \mathbf{V}_{\mathbf{u}} \cdot \frac{\partial \mu}{\partial \mathbf{u}} d \mathbf{u}-\frac{m}{T} \int \mathbf{J} \cdot \nabla\left[\frac{1}{2}\left(\mathbf{u}-\mathbf{v}_{0}\right)^{2}\right] d \mathbf{u}-\frac{m}{T} \int \mathbf{J}_{0} \cdot \mathbf{F} d \mathbf{u} .
$$

This entropy production rate contains three contributions. The first one is due to diffusion in $\mathbf{u}$-space and is proportional to the product of the mesoscopic probability current $f \mathbf{V}_{\mathbf{u}}$ and the derivative with respect to particle velocity $\mathbf{u}$ of the nonequilibrium chemical potential $\mu(\mathbf{r}, \mathbf{u}, t)$ per unit mass

$$
\mu(\mathbf{r}, \mathbf{u}, t)=\frac{k_{B} T}{m} \ln |f(\mathbf{r}, \mathbf{u}, t)|+\frac{1}{2}\left(\mathbf{u}-\mathbf{v}_{0}\right)^{2} .
$$


The second contribution originates from the product between the gradient of the local kinetic energy of the particles $\frac{1}{2}\left(\mathbf{u}-\mathbf{v}_{0}\right)^{2}$ (see also (de Groot \& Mazur, 1984)), and the relative current $\mathbf{J}=(\mathbf{u}-\mathbf{v}) f$, where $\mathbf{v}$ represents the average velocity field of the particles,

$$
\rho \mathbf{v}(\mathbf{r}, t)=m \int \mathbf{u} f(\mathbf{r}, \mathbf{u}, t) d \mathbf{u} .
$$

Finally, the third contribution to the entropy production rate involves the product of the relative current $\mathbf{J}=\left(\mathbf{u}-\mathbf{v}_{0}\right) f$ times the force $\mathbf{F}=d \mathbf{v}_{0} / d t$ arising from the dependence on time of the external velocity field $\mathbf{v}_{0}(\mathbf{r}, t)$.

A linear law for the mesoscopic current $f \mathbf{V}_{\mathbf{u}}$ in terms of the force was proposed, and when substituted into Eq. (16), it yields the Fokker-Planck equation

$$
\frac{\partial f}{\partial t}+\nabla \cdot(\mathbf{u} f)=\frac{\partial}{\partial \mathbf{u}} \cdot\left\{\underline{\beta} \cdot\left(\mathbf{u}-\mathbf{v}_{0}\right) f-\underline{\zeta} \cdot \mathbf{F} f+\mathbb{D}_{v} \cdot \frac{\partial f}{\partial \mathbf{u}}\right\} .
$$

The postulation of a linear law scheme necessarily introduces the Onsager coefficients $\beta, \underline{\zeta}$ and $\underline{\epsilon}$. The diffusion coefficient in velocity space $\mathbb{D}_{v}$, is related to $\underline{\beta}$ and $\underline{\epsilon}$ through the expression

$$
\mathbb{D}_{v}=\frac{k_{B} T}{m}\left[\underline{\beta}-\underline{\epsilon} \cdot \nabla \vec{v}_{0}\right]
$$

In Eq. (23), $\beta$ is the friction coefficient that usually appears when describing Brownian motion in velocity space (Santamaría Holek, 2005; 2001), and corresponds to what is referred as a direct effect in nonequilibrium thermodynamics (de Groot \& Mazur, 1984). The cross coefficient $\underline{\zeta}=\rho_{p} / \rho_{f} \underline{1}$ is related to inertial effects and $\rho_{p}$ and $\rho_{f}$ denote the particle and host fluid densities, and $\underline{1}$ is the unit tensor. The tensor $\underline{\epsilon}$ constitutes an important result of this analysis. It corresponds to a cross effect term proportional to the gradient of the imposed velocity flow: $\nabla \mathbf{v}_{0}$.

The expressions for $\underline{\epsilon}$ and $\zeta$ were obtained by using the generalized Faxén theorem giving the force experienced by a particle of arbitrary shape into a fluid under non-stationary flow conditions (Mazur \& Bedeaux, 1974; Santamaría Holek, 2005). For spherical particles at low Reynolds numbers, $\underline{\epsilon}=\epsilon \underline{1}, \epsilon$ is given by

$$
\epsilon=\frac{1}{6} \frac{m}{k_{B} T} a^{2} \beta_{0}^{2} k_{\omega}
$$

where $k_{\omega}=\left(1+2 a \alpha_{\omega}\right), \alpha_{\omega}=\sqrt{-i \omega / v}$ is the inverse viscous penetration length of the host fluid and $v$ its kinematic viscosity. Finally, $\beta_{0}=6 \pi \eta a / m$ is the Stokes friction coefficient where $\eta$ is the dynamic viscosity of the host fluid.

The main fact to stress here is that the fluctuation term in the Fokker-Planck equation (23) contains the diffusion coefficient in velocity space $\mathbb{D}_{v}$, Eq. (24), that is similar to the one obtained through kinetic theory, see Eq. (8). This coefficient contains the expected thermal contribution $k_{B} T \beta / m$ and, more interesting, a non-thermal contribution coming from the cross effect coupling the diffusion of probability in configuration and velocity subspaces: $\frac{1}{6} a^{2} \beta_{0}^{2} \nabla \vec{v}_{0}$. This particular dependence of $\mathbb{D}_{v}$ leads to a modification of the spatial diffusion coefficient $\mathbb{D}(\mathbf{r}, t)$ of the Brownian particles which is in agreement with experimental and simulation results (Pine, 2005; Sarman, 1992). However, before discussing this point, some comments on the diffusion or Smoluchowski equation are in turn. 


\subsection{Diffusion in the presence of shear flow}

The Smoluchowski equation can be derived from Eq. (23) by restricting the description to the long-time diffusion regime, that is, when $t>>\beta_{i j}^{-1}$.

In references (Santamaría Holek, 2005; 2009; 2001), the Smoluchowski equation was obtained by calculating the evolution equations for the first moments of the distribution function. These equations constitute the hydrodynamic level of description and can be obtained through the Fokker-Planck equation. The time evolution of the moments include relaxation equations for the diffusion current and the pressure tensor, whose form permits to elucidate the existence of inertial (short-time) and diffusion (long-time) regimes. As already mentioned, in the diffusion regime the mesoscopic description is carried out by means of a Smoluchowski equation and the equations for the moments coincide with the differential equations of nonequilibrium thermodynamics.

The procedure is enterily similar to that followed in kinetic theory (McLennan, 1989). In particular, after assuming that stresses relax rapidly enough, the resulting Smoluchowski equation is

$$
\frac{\partial \rho}{\partial t}=-\nabla \cdot\left(\rho \mathbf{v}_{0}+\zeta \underline{\xi} \cdot \vec{F}\right)+\nabla \cdot(\mathbb{D} \cdot \nabla \rho),
$$

where the effective diffusion coefficient is defined according to the relation

$$
\mathbb{D}=\frac{k_{B} T}{m} \underline{\xi} \cdot\left(\underline{\mathbf{1}}-\underline{\xi} \cdot \nabla \mathbf{v}_{0}\right)^{s}-\frac{1}{6} a^{2} \beta_{0}^{2} k_{\omega} \underline{\xi} \cdot\left(\underline{\xi} \cdot \nabla \mathbf{v}_{0}\right)^{s} .
$$

Here $\underline{\xi}$ is the mobility tensor satisfying $\underline{\xi} \cdot \underline{\beta}=\underline{\mathbf{1}}$. Again, this diffusion coefficient contains two contributions, one depending on the temperature $T$ of the fluid and another one independent from it and proportional to the velocity gradient of the imposed external flow. Therefore, the first contribution is related to the usual thermal Brownian motion whereas the last one is related to non-thermal effects. Notice also that the Smoluchowski equation (26) also contains the usual convective term $\nabla \cdot\left(\rho \mathbf{v}_{0}\right)$.

Thus, the effect of the flow on diffusion is to introduce a new contribution term in the diffusion coefficient arising from spatial inhomogeneities of the velociy field. As a consequence, the flow also induces anisotropy in the Brownian motion. This can be seen from the fact that even if the host fluid is assumed isotropic and the particles spherical, implying $\beta=\beta \underline{1}$, the effective diffusivity is a tensor proportional to $\nabla \mathbf{v}_{0}$. It is convenient to mention that this non-thermal contribution, known as the shear-induced diffusion effect, is proportional to the size (surface) of the particle and breaks-down the fluctuation-dissipation relation of the Smoluchowski equation. The presence of a nonequilibrated bath therefore modifies the thermal energy available to the system in order to perform fluctuations. As shown before, similar results are obtained from kinetic theory (Rodríguez, 1983). These results suggest that the fluctuation dissipation relation used in Langevin-like approaches should be modified accordingly for appreciably large Reynolds numbers. Comparison of these results with other theories like, for instance, extended irreversible thermodynamics could be of interest.

\subsection{Classical simulation results on diffusion in the presence of shear flow}

It is convenient to emphasize that nonequilibrium molecular dynamics simulations with Lennard-Jones pure fluids and mixtures under strong shear flow conditions (Sarman, 1992), lead to a dependence of the self-diffusion tensor on the shear rate.

The results on diffusion reported show that the components of the diffusion tensor start increasing or decrasing linearly as a function of the shear rate $\dot{\gamma}$, (Sarman, 1992), in 
agreement with the results obtained with kinetic theory and mesoscopic nonequilibrium thermodynamics. For larger values of $\dot{\gamma}$, a quadratic contribution seems to appear and modifies the initial tendency. In general, diagonal elements are positive whereas the non-diagonal elements are negative. However, despite the sign of each element of the diffusion tensor, the authors emphasize that the dependence on $\dot{\gamma}$ and the obtained anisotropy for the self-diffusion tensor evidences a break-down of the Curie principle and of the local equilibrium hypothesis of linear irreversible thermodynamics, thus implying an anisotropic kinetic energy distribution induced by the shear flow.

These observations pose an interesting question because the results reported in Refs. (Santamaría Holek, 2005; 2009; 2001) were obtained under the assumption of local equilibrium in phase space, that is, at the mesoscale. It seems that for systems far from equilibrium, as those reported in (Sarman, 1992), the validity of the fundamental hypothesis of linear nonequilibrium thermodynamics can be assumed at the mesoscale. After a reduction of the description to the physical space, this non-Newtonian dependedence of the transport coefficients on the shear rate appears. This point will be discussed more thoroughly in the following sections when analyzing the formulation of non-Newtonian constitutive equations.

\subsection{The shear-induced diffusion effect and the transition to irreversibility}

One of the most beautiful results manifesting the effect of flow on diffusion is the shear-induced diffusion effect, which is the quantitative manifestation of the transition to irreversibility in a set of particles obeying the classical reversible equations of motion.

Recently, this effect was studied in a series of experiments and numerical simulations were the transition from a dynamical reversible behavior to a dynamical irreversible chaotic behavior of a suspension of non-Brownian particles was directly observed (Drazer, 2002; Pine, 2005). The importance of these experiments lies in the fact that they shed light on the origin of the thermodynamic irreversibility and its relation to the chaotic dynamics of a system (Guasto \& Gollub, 2007; Pine, 2005).

The experiments were performed with semi-diluted suspensions of spherical particles with diameters $d \simeq 0.2 \mathrm{~mm}$. The host fluid had the same density as the particles and was highly viscous. The suspension was contained in a cylindrical Couette cell which was taken out from equilibrium by applying an oscillating shear flow $\dot{\gamma} \cos (\omega t)$ with dimensionless amplitude $\gamma$ and rate $\dot{\gamma}=\omega \gamma$, being $\omega$ the characteristic frequency of the oscillation. The Reynolds number of the flow was always much smaller than one, thus avoiding turbulent effects. For small oscillation amplitudes $\gamma$ the motion of the particles was reversible, that is, after each period the particles came back to the original position. On the contrary, when increasing the oscillation amplitude $\gamma$ the trajectories of the particles became chaotic implying that their reversible behavior was lost. From the thermodynamic point of view, it was interesting to know that the motion of the particles was characterized by means of the mean square displacement $\left\langle\Delta x^{2}\right\rangle$, which scaled with time in the form: $\left\langle\Delta x^{2}\right\rangle \sim 2 d^{2} \dot{\gamma} t$, thus implying that the effective diffusivity scales with $D \sim d^{2} \dot{\gamma}$. Computer simulations using Stokesian dynamics (Bossis \& Brady, 1989; Pine, 2005) modeled the experiment and determined a positive Lyapunov exponent of particle trajectories in phase space (Pine, 2005), thus implying that particle dynamics is chaotic. The transition was characterized by giving quantitative values for the critical strain amplitude at which it occurs.

Different theoretical descriptions were proposed to explain this effect. One of these models is based on a Smoluchowski equation in which the diffusivities have been constructed by analyzing the temporal behavior of the correlation of the position of the particles as 
obtanied from simulations (Seriou \& Brady, 2004). However, this description does not impose new thermodynamic postulates but it gives good quantitative results, though it lacks of a deeper fundamental basis. This fundamental analysis can be given in terms of mesocopic nonequilibrium thermodynamics (Santamaría Holek, 2009).

The explanation of the effect was formulated for a set of $N$ particles described through a multivariate probability distribution. After deriving the corresponding Fokker-Planck equation along the lines indicated above, the description was reduced to the case of a single tagged particle in an effective medium composed by the host fluid and the remaining particles. The main result was a Smoluchowski equation for the single particle distribution function $\rho(\mathbf{r}, t)$ entirely similar to Eq. (26), with an effective diffusion coefficient of the form (27). However, in the referred case the diffusion tensor depends on position and time, and on the fraction of volume $\phi=N V_{p} / V$ occupied by the particles in the sample. $V_{p}$ and $V$ are the volume of a particle and the total volume. These dependencies arise when reducing the $\mathrm{N}$-particle description to the single-particle one, a process that involves averaging over positions and (Santamaría Holek, 2009) velocities of the remaining particles (Santamaría Holek, 2009).

For sufficiently large particles (with vanishing thermal contribution) it was possible to show that the mean square displacement is of the form

$$
\left\langle r^{2}\right\rangle \sim \frac{1}{3} g_{12}(\phi) d^{2} \dot{\gamma} t
$$

expressed in terms of the number of cycles $n$ hence $t=2 \pi n / \omega$; thus $\left\langle r^{2}\right\rangle \sim \frac{2 \pi}{3} g_{12}(\phi) d^{2} \gamma_{0} n$, which has the same scaling relation for the mean square displacement than that reported in the experiments in Ref. (Pine, 2005). Here, the dimensionless factor $g_{12}(\phi)$ depending on the volume fraction $\phi$ is a consequence of the existence of hydrodynamic interactions between particles.

\subsection{Lattice Boltzmann simulations}

Lattice Boltzmann simulations using the D2Q9 model (Aidun \& Ding, 1998; Ladd, 1994) were also performed to study the transition to irreversibility in sheared suspensions of non-Brownian particles (Santamaría Holek, 2009). This method was used because it esentially controls the evolution in time of the host fluid without thermal fluctuations, thus giving in a natural way the hydrodynamic interactions that, for large shear rates and particle concentrations, dominate the dynamics of the system. Particle interactions were implemented with the method proposed in Ref. (Ladd, 1994). The numerical simulations were carried out in a cavity with the bounce-back boundary condition, which consists in reversing the incoming particle distribution function after the stream process.

From these simulations it was observed that the effective diffusion of the particles can be enhanced by increasing the particle concentration $\phi$ or by increasing the Reynolds number $R e$, even at small values of the Re. The transition to irreversibility was characterized through a power spectrum of particle trajectories, as shown in Fig. 1, where the trajectory of one of the sixteen particles is shown. In Fig. 1a, one can appreciate that the particle describes regular motion and even though in Fig. $1 \mathrm{~b}$ we can notice some regularity in the motion. However, it is clear that hydrodynamic interactions induce correlations between all particles giving rise to new dissipation modes in the dynamics. By increasing the Reynolds number above its critical value (corresponding to the critical value of the strain amplitude) the motion becomes chaotic, as shown in Fig. 1e. Correspondingly, the spectrum shows (Fig. 1f) that almost all frequencies contribute with the same amplitude, thus suggesting the stochastization of the motion. 

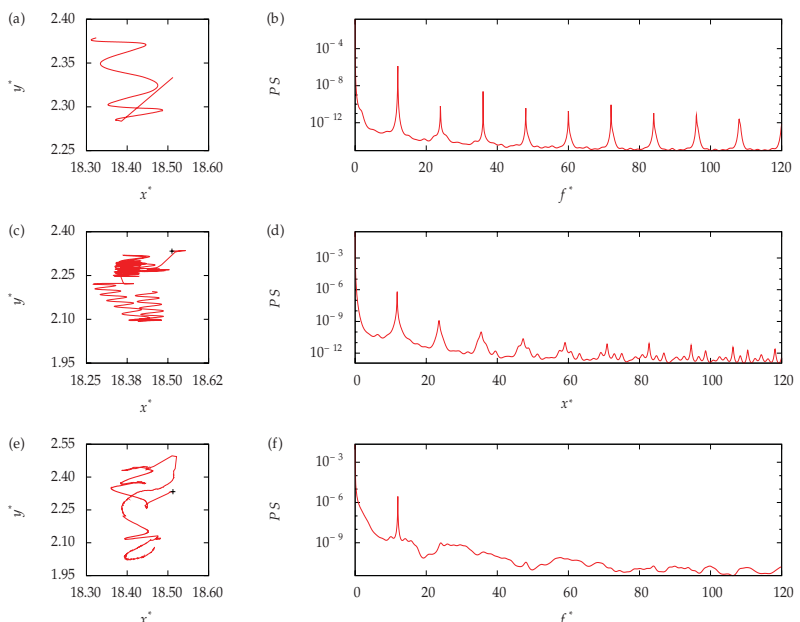

Fig. 1. Trajectories and power spectra of the same particle with equal initial conditions for (a-b) $R e=0.01$ and (c-d) $R e=0.07$ and (e-f) $R e=0.08$ with a volume fraction of $\phi=0.14$ and dimensionless frquency $f^{*}=10.0$. The figure is a courtesy of Dr. G. Barrios.

To close this section, we want to emphasize that the explanation of the experiments and simulations on shear-induced diffusion shows the validity of the theoretical approach and its importance, because it was shown that the shear-induced diffusion and the transition to irreversibility are fully compatible with the thermodynamic irreversibility as obtained from the second principle and the generalized Gibbs entropy postulate.

\section{Constitutive relations}

The main question related to the thermodynamics in presence of flow is the formulation of constitutive relations between density currents and thermodynamic forces. In linear irreversible thermodynamics (de Groot \& Mazur, 1984; Kondepudi \& Prigogine, 1999) the constitutive relations are linear. Although this approximation is valid for a large number of systems, there are also many of them in which these linear relationships are not valid. A classical example of this are chemical reactions, where the currents are sometimes not linearly related to the forces, like in the mass action law (de Groot \& Mazur, 1984; Kondepudi \& Prigogine, 1999).

Flowing systems are also typical examples in which this linear behavior is broken. This is the case of rheology, the study of viscosity of complex fluids, that is, of fluids with internal structure that exhibit a combination of viscous and elastic behavior under strain (Beris \& Edwards, 1994; Doi \& Edwards, 1998). Examples of such fluids are polymer solutions and melts, oil and toothpaste, among many others.

There are several approaches leading to the formulation of these non-linear relationships. Unfortunately it is not possible to summarize them here because the field is very rich in the variety of systems and behaviors. Consequently, such a summary lies beyond the scope of this study. An excellent and detailed review is given in Ref. (Beris \& Edwards, 1994). However, let us mention that many attempts have been made to bring together continuum mechanics theories and molecular models in order to formulate appropriate constitutive equations for 
polymer solutions (Beris \& Edwards, 1994; Doi \& Edwards, 1998). One of the central aspects of this research is the incorporation of microscopic theories such as those by Zimm or Rouse (Doi \& Edwards, 1998) in constitutive relations. However, these attempts are usually valid only in the dilute regime, although the proposed rheological equations are often evaluated quantitatively and qualitatively to describe concentrated colloidal suspensions or polymer solutions (James, 1972). From a phenomenological point of view, the first model attempting to describe the hydrodynamic behavior of complex fluids was proposed by Maxwell and describes what is now known as Maxwell materials (Meyers \& Chawla, 2009). Other type of materials are Kelvin-Voigt and Oldroyd materials.

The main difference between constitutive relations for simple and complex fluids lies in the fact that simple fluids satisfy the linear relationships between the stress tensor $\underline{\sigma}(\mathbf{r}, t)$ and the strain or deformation tensor $\underline{\theta}(\mathbf{r}, t)$ or the velocity gradient $\nabla \mathbf{v}$. In contrast, complex fluids have to incorporate a relaxation term for the stresses and a non-linear dependence on the strain or velocity gradient tensors.

\subsection{Simple viscoelastic materials}

For simple viscoelastic materials, the main characteristic of the constitutive relations is the presence of a time derivative of the stress tensor, associated to a relaxation process. In the case of an incompressible Maxwell material, the constitutive relation is given through the evolution equation

$$
\tau \frac{\partial}{\partial t} \underline{\sigma}+\tau(\mathbf{v} \cdot \nabla \underline{\sigma})_{\text {cont }}=-\underline{\sigma}+\eta\left(\nabla \mathbf{v}+\nabla \mathbf{v}^{T}\right)
$$

where $\tau$ is a relaxation time of the material and the contravariant convective term is given by the second order tensor $\left\{(\mathbf{v} \cdot \nabla \underline{\sigma})_{\text {cont }}\right\}_{i j}=v_{k} \nabla_{k} \sigma_{i j}-\sigma_{i k} \nabla_{k} v_{j}-\sigma_{k j} \nabla_{k} v_{i}$. This convective term has been proposed by Oldroyd in order to satisfy the material invariance condition (Beris \& Edwards, 1994). As mentioned before, the last term of the equation is often expressed in terms of the rate of the deformation tensor $\underline{\theta}$. This model is related to microscopic approaches where the molecules are modeled as dumbells (Hinch, 1994; Rallison \& Hinch, 1988). Clearly, this equation is coupled to the momentum equation and can be solved for many types of flows. Averaged over volume, Maxwell's model is frequently expressed in the more simple form

$$
\frac{d}{d t} \underline{\hat{\sigma}}=-\frac{1}{\tau} \underline{\hat{\sigma}}+\frac{\eta}{\tau} \frac{d}{d t} \underline{\hat{\theta}}
$$

where $\hat{\sigma}$ and $\hat{\theta}$ are now time dependent quantities only. Assuming that the rate of deformation is constant $d \underline{\hat{\theta}} / d t=\underline{\dot{\theta}}$, one then obtains that the constitutive relation for a simple viscoelastic fluid is of the form

$$
\underline{\hat{\sigma}}=\eta \underline{\dot{\theta}}\left(1-e^{-t / \tau}\right)
$$

that is, after applied an external force inducing the deformation of the material, the stress tensor relaxes until reaching a stationary value. When an oscillatory shear is applied on the system then one obtains that the viscoelastic response of the fluid is characterized by a frequency dependent viscosity.

\subsubsection{Non-Newtonian materials}

Very frequently, complex fluid manifest non-linear constitutive relations between the stress tensor and the velocity gradient. These relations can be a consequence of the fact that the relaxation time dependens on a scalar function of the deformation tensor like in the FENE models (Hinch, 1994; Rallison \& Hinch, 1988) or of the stress tensor, like in the case of the 
so-called modified Maxwell models (Beris \& Edwards, 1994), that is, $\tau=\tau(\operatorname{tr}[\underline{\sigma}]$ ) where the dependence on the stress tensor is usually introduced via the invariants of $\underline{\sigma}$, for example, through $\operatorname{tr} \underline{\sigma}$. As a consequence of this, the evolution equation for the fluid becomes of the form

$$
\frac{\partial}{\partial t} \underline{\sigma}+(\mathbf{v} \cdot \nabla \underline{\sigma})_{\text {cont }}=-\frac{\underline{\sigma}}{\tau(\operatorname{tr}[\underline{\sigma}])}+\frac{\eta}{\tau(\operatorname{tr}[\underline{\sigma}])}\left(\nabla \mathbf{v}+\nabla \mathbf{v}^{T}\right) \text {. }
$$

Frequently, non-Newtonian materials show a power-law behavior of the viscosity as a function of the shear rate $\dot{\gamma}$ in which the exponent is a free parameter. An example of this is the following model for the relaxation time (Apelian, 1988)

$$
\tau(\operatorname{tr}[\underline{\sigma}])=\frac{\tau_{0}}{1+\left\{h\left(\tau_{0}\right) \operatorname{tr}[\underline{\sigma}]\right\}^{\alpha-1}},
$$

where $\tau_{0}$ is a charateristic relaxation time and $h$ is a function that gives the correct dimensions to the formula. As a consequence of this type of dependencies, it is ussually obtained that the viscosity of the fluid and the normal stress difference $\Psi$ depend on the shear rate in the form

$$
\eta \sim \dot{\gamma}^{k(\alpha)} \quad \text { and } \quad \Psi \sim \dot{\gamma}^{2},
$$

where in general the exponent of the shear rate $k$ depends on $\alpha$.

These examples illustrate that viscoelastic and non-Newtonian materials do not respond instantaneously to the shear stresses applied on them and its response is not linear with respect to those stresses.

\subsection{Mesoscopic constitutive relations for polymer solutions}

Nonlinear constitutive relations for complex fliuds can also be obtained by using MNET (Málaga, 2006). As described in Section II, the description was given in terms of a probability density accounting for the state of the system through the position $\mathbf{r}$ and instantaneous velocity $\mathbf{u}$ of the molecules. The deformation of each molecule is modeled by introducing a local vector parameter $\mathbf{R}$.

Following the scheme of MNET, a Fokker-Planck equation was obtained from which a coarse-grained description in terms of the hydrodynamic equations was derived in turn. Molecular deformation and diffusion effects become coupled and a class of non-linear constitutive relations for the kinetic $\mathbb{P}^{k}$ and elastic parts $\mathbb{P}^{E}$ of the stress tensor are obtained. The expression for the stress tensor can be written in terms of dimensionless quantities like

$$
\underline{\sigma}=\mathbb{P}^{k}+\mathbb{P}^{E}
$$

Each contribution can be obtained from its own evolution equation. The kinetic part of the stress tensor is governed by the equation

$$
\frac{d \mathbb{P}^{k}}{d t}+2\left[\mathbb{P}^{k} \cdot(\underline{\beta}+\nabla \mathbf{v})\right]^{s}=2 \frac{k_{B} T}{m} \rho\left(\underline{\beta}-\mathbb{L}_{u r} \cdot \nabla \mathbf{v}_{0}\right)^{s}
$$

where the superscript ${ }^{s}$ stands for the symmetric part of a tensor, $m$ is the mass of the molecule, $\beta$ is the the friction tensor of the particles proportional to the corresponding Stokes coefficient (Santamaría Holek, 2001), and $\mathbb{L}_{u r}$ is an Onsager coefficient. Notice that in contrast with Eqs. (29) and (32), where the relaxation time is scalar, Eq. (36) contains a matrix of the relaxation times $\underline{\tau} \equiv \underline{\beta}+\nabla \mathbf{v}$. This means that in contrast with the usual phenomenological 


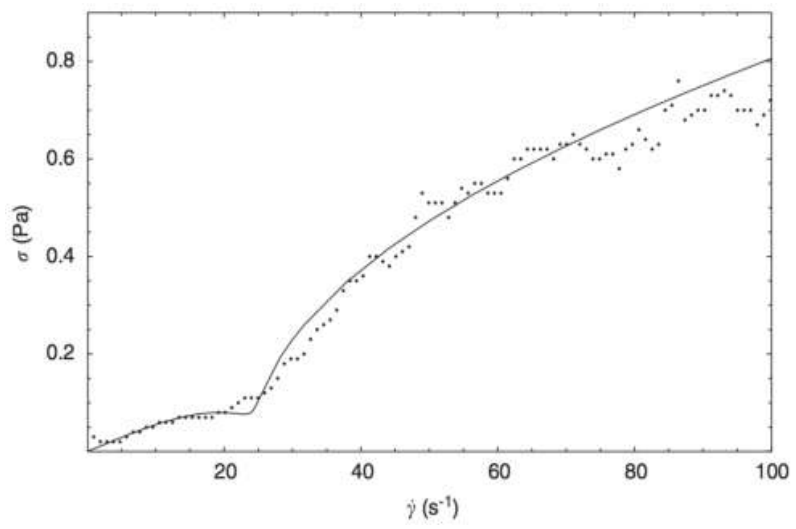

Fig. 2. Experimental data (points) for the non-Newtonian shear stress versus the shear rate of a dilute solution of wormlike micelles (Delgado \& Castillo, 2005; 2007). Fit of the data using the isotropic friction model (line) in Eqs. (35), (36) and (38). Notice that the dependence of the effective viscosity as a function of the shear rate is not simply a power law. Figure taken from Ref. (Málaga, 2006).

relations such as (29) and (32), the relaxation time of (36) has an explicit relation in terms of the friction coefficients of the particles in the host fluid.

For a FENE-type model with nonlinear elastic force of the dumbell, the restituting elastic force of a molecule can be represented in the form: $\mathbf{F}=-\xi_{0} F_{0} \mathbf{R}$, where $\xi_{0}$ is the characteristic spring restitution coefficient per unit mass and $F_{0}=L^{2} /\left(L^{2}-\operatorname{tr} \mathbb{A}\right)$ with $L$ the maximum length of the polymer. The evolution equation for the elastic contribution is given in terms of the deformation or conformational tensor $\mathbb{A}=\langle\mathbf{R} \mathbf{R}\rangle$, which is now related to the elastic part of the stress tensor by the formula $\mathbb{P}^{E}=-\xi_{0} F_{0} \mathbb{A}$. The equation is

$$
\frac{d \mathbb{A}}{d t}-2[\mathbb{A} \cdot \nabla \mathbf{v}]^{s}=2\left\{\mathbb{L}_{R R} \cdot\left[\frac{k T}{m} \mathbb{I}-\langle\mathbf{F R}\rangle\right]\right\}^{s}+2\left[\mathbb{L}_{R r} \cdot\left(\nabla \mathbf{v} \cdot \nabla \mathbf{v}^{T}\right) \cdot \mathbb{A}\right]^{s},
$$

where $\mathbb{L}_{R r}$ and $\mathbb{L}_{R R}$ are Onsager coefficients characterizing the mobility and the coupling between the deformation of the molecule and drag forces, respectively. The mesoscopic formalism leads naturally to the Oldroyd type derivative that maintains material frame independence (Málaga, 2006). Eqs. (36) and (37) have similar features as those indicated by the Maxwell and the modified Maxwell models already discussed.

These equations were applied to describe different situations. For isotropic friction, the dynamics of dumbell-like molecule solutions under simple shear conditions was analyzed to obtain a correction to the usual FENE models that arises through the kinetic contribution to the stress tensor. A second application for non-constant friction coefficient $\beta$ lead to the evolution equation

$$
\frac{d \mathbb{A}}{d t}-\mathbb{A} \cdot \nabla \mathbf{v}_{0}-\nabla \mathbf{v}_{0}^{T} \cdot \mathbb{A}=\frac{16}{9 \sqrt{t r \mathbb{A}}} \frac{1}{D e}\left(\mathbb{I}-\xi_{0} F_{0} \mathbb{A}\right)
$$

where $D e=\beta \dot{\gamma} / \xi_{0}$ is the Deborah number. As a consequence of this equation, a non-linear relation between the elastic part of the stress tensor $\mathbb{P}^{E}$ and the shear rate $\dot{\gamma}$ appears. This relation was used to describe satisfactorily experiments with dilute miscellar solutions in water (Málaga, 2006), see Fig. 2. 


\section{Nonequilibrium equations of state}

One interesting aspect of the previous discussion is that the expressions for the normal as well as for the diagonal elements of the stress tensor $\underline{\sigma}$ or, more generally, the pressure tensor $\mathbb{P}$ are functions of the applied velocity gradient or the strain rate. In this sense, these relations represent what is known as nonequilibrium equations of state. It was shown rigorously that at mesoscopic level, the chemical potential becomes modified by the external flow in a term proportional to its local kinetic energy, see Eq. (21).

In this section we want to address how the presence of flow may lead to the existence of these nonequilibrium equations of state at macroscopic level. For convenience we will only consider the case of particle suspensions (Santamaría Holek, 2005), that is, we will not consider the internal degrees of freedom of each molecule, as we did for the case of polymer solutions.

Let us recall that the evolution equation for the kinetic part of the pressure tensor $\mathbb{P}^{k}$ was given by equation (36). For times $t \gg \beta^{-1}$ the particles enter the diffusion regime governed by the corresponding Smoluchowski equation. In this regime, assuming a stationary process, the following constitutive relation for $\mathbb{P}^{k}$ was obtained

$$
\mathbb{P}^{k} \simeq \frac{k_{B} T}{m} \rho \underline{1}-\left[\left(\underline{\eta}_{B}+\underline{\eta}_{H}\right) \cdot \nabla \vec{v}_{0}\right]^{s},
$$

where the Brownian $\left(\underline{\eta}_{B}\right)$ and hydrodynamic $\left(\underline{\eta}_{H}\right)$ viscosity tensors are defined by

$$
\underline{\eta}_{B} \equiv \frac{k_{B} T}{m} \rho \underline{\beta}^{-1} \quad \text { and } \quad \underline{\eta}_{H} \equiv \frac{k_{B} T}{m} \rho \underline{\beta}^{-1} \cdot \underline{\epsilon} .
$$

For spherical particles, the friction coefficient $\beta$ can be identified with the Stokes friction coefficient $\underline{\beta} \simeq \beta \underline{1}$. Two cases are of interest.

\subsection{Oscillatory flows in the creeping flow approximation}

When the oscillatory flow is slow enough, by using Faxen's theorem it was shown that the friction coefficient depends on the frequency $(\omega)$ in the form (Mazur \& Bedeaux, 1974)

$$
\beta=\beta_{0}\left(1+a \alpha_{\omega}\right),
$$

where $\alpha_{\omega}=\sqrt{-i \omega / \nu(\omega)}$, is the inverse viscous penetration length of the host fluid with kinematic viscosity $v(\omega)$. The expression for $\epsilon$ is given through Eq. (25). Using these results it was found that the expressions for the Brownian $\underline{\eta}_{B}(\omega)$ and hydrodynamic $\underline{\eta}_{H}(\omega)$ viscosities are

$$
\underline{\eta}_{B} \simeq \frac{k_{B} T}{m \beta_{0}} \rho\left[1-\Delta_{\omega}(\tau \omega)^{\delta_{\omega}}\right] \quad \text { and } \quad \underline{\eta}_{H} \simeq \frac{1}{12} \beta_{0} a^{2} \rho\left[1+\Delta_{\omega}(\tau \omega)^{\delta_{\omega}}\right],
$$

where $\Delta_{\omega}$ and $\delta_{\omega}$ are a scaling factor and an exponent that in general may depend on the particle volume fraction $\phi$. For viscoelastic fluids the expressions corresponding to the normal stress difference and the non-diagonal elements of the pressure tensor are

$$
\begin{aligned}
P_{x x}-P_{y y} & \simeq \beta_{0}^{-1}\left[\underline{\eta}_{B}(\omega)+\underline{\eta}_{H}(\omega)\right] \dot{\gamma}^{2}, \\
P_{x y} & \simeq \beta_{0}^{-1}\left[\underline{\eta}_{B}(\omega)+\underline{\eta}_{H}(\omega)\right] \dot{\gamma} .
\end{aligned}
$$

This relation suggests that the normal stresses of simple viscoelastic fluids depend on the shear rate $\dot{\gamma}$, that is, are modified by the flow conditions $P_{i i} \propto \dot{\gamma}^{2}$, see Eq. (34). 


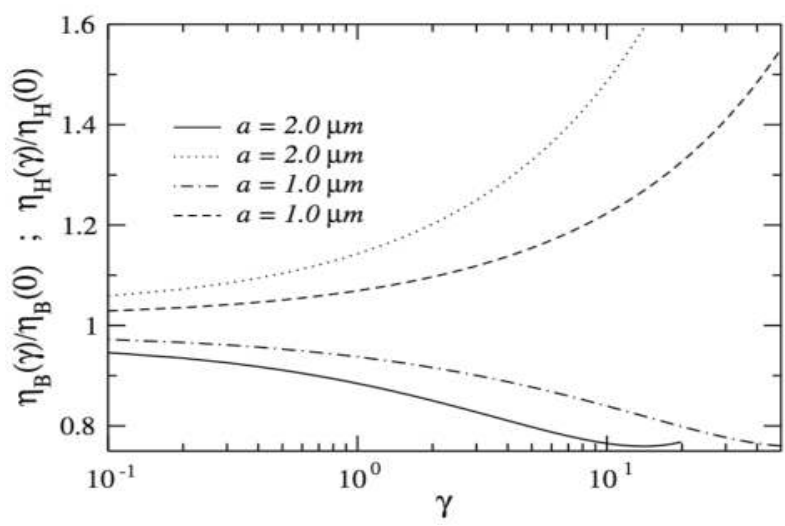

Fig. 3. Brownian (solid and dash-dotted lines ) and hydrodynamic (dashed and dotted lines) viscosities in terms of the shear rate for different values of the radius of the Brownian particle. The figure shows the shear thinning and shear thickening effects associated to the Brownian and hydrodynamic contributions, respectively. The values of the parameters we have used are $\beta_{0}=10^{6} \mathrm{~s}^{-1}, D_{0}=10^{6} \mathrm{~cm}^{2} \mathrm{~s}^{-1}$ and $\rho=10^{-2} \mathrm{gcm}^{-3}$. Figure taken from Ref. Santamaría Holek (2005).

\subsection{Non-Newtonian behavior}

When the applied shear is large enough, the friction coefficient becomes a function of the shear rate in the form (Santamaría Holek, 2005)

$$
\underline{\beta}^{-1}=\beta_{0}^{-1}\left(\underline{1}-\mathbb{M} a \alpha_{\dot{\gamma}}\right)
$$

where $\alpha_{\dot{\gamma}}=\sqrt{\dot{\gamma} / \nu(\omega)}$, is the inverse viscous penetration length due to the presence of a large shear flow and $\mathbb{M}$ is a geometrical factor depending on the boundary conditions over the surface of the particle. Using the corresponding expression for $\epsilon(\dot{\gamma})$ it is found that in the concentrated case, the non-Newtonian expressions for the Brownian and hydrodynamic viscosities are

$$
\underline{\eta}_{B} \simeq \frac{k_{B} T}{m \beta_{0}} \rho\left[1-M_{x x} \Delta_{\gamma}(\tau \dot{\gamma})^{\delta_{\gamma}}\right] \quad \text { and } \quad \underline{\eta}_{H} \simeq \frac{1}{12} \beta_{0} a^{2} \rho\left[1+M_{x x} \Delta_{\gamma}(\tau \dot{\gamma})^{\delta_{\gamma}}\right] .
$$

where, as in the previous case, the scaling factor $\Delta_{\gamma}$ and the exponent $\delta_{\gamma}$ are $\phi$ dependent. For the present case, the expressions corresponding to the normal stress difference and the non-diagonal elements of the pressure tensor are

$$
\begin{aligned}
P_{x x}-P_{y y} & \simeq-\frac{M_{x y}}{3} \beta_{0}^{-1} a^{2} \rho \Delta_{\gamma} \tau^{\delta_{\gamma}} \dot{\gamma}^{1+\delta_{\gamma}}, \\
P_{x y} & \simeq \beta_{0}^{-1}\left[\underline{\eta}_{B}(\dot{\gamma})+\underline{\eta}_{H}(\dot{\gamma})\right] \dot{\gamma} .
\end{aligned}
$$

These results, together with those for pure viscoelastic fluids clearly suggest the possibility of the existence of non-equilibrium equations of state, that is, equations of state that are modified by the presence of the flow. This possibility will be analyzed more thoroughly in the following sections. 


\section{Nucleation in the presence of flow}

One of the interesting applications where the influence of flow on transport coefficients and on the constitutive and state equation is relevant, is the nucleation process. The effect of flow on crystallization is important because it often occurs in the presence of mechanical processing of the melt, such as extrusion, shearing, or injection, all these factors may affect the process drastically.

Despite the various existing approaches to the problem, we will restrict the presentation to that based on MNET (Gadomski, 2005; 2008; Reguera \& Rubi, 2003b). In this approach the influence of a shear flow on the process of nucleation is analyzed through the derivation of a Fokker-Planck equation for the probability distribution that depends on size. This is done for a metastable phase subjected to a stationary flow. In addition to the correction of the diffusion coefficient, it was shown that to the flow also introduces modifications to the nucleation rate. By considering a metastable phase in which the emerging clusters, liquid droplets or crystallites are embedded, the description is given in terms of the probability distribution $f(\mathbf{r}, \mathbf{u}, n, t)$, where now $n$ denotes a cluster of $n$ molecules. Following a scheme similar to that of Section 2, after proposing the Gibbs equation the authors assume that the local equilibrium distribution [see Eqs. (17) and (19)] has the form

$$
f_{l e q}(\mathbf{r}, \mathbf{u})=\exp \left\{\left[\mu_{B}-\Delta G(n)+\frac{1}{2} m(n)\left(\mathbf{u}-\mathbf{v}_{0}\right)^{2}\right] / k_{B} T\right\}
$$

The description given in Ref. (Reguera \& Rubi, 2003b) is completely general since the particular for of the free energy difference is not specified. However, to fix ideas, one may assume that $\Delta G(n)$ is given by the expression of the classical nucleation theory

$$
\Delta G(n)=-n \Delta \mu+v_{a}^{*} \tilde{\sigma} n^{2 / 3}
$$

where $\Delta \mu$ is the difference in chemical potential between the solid and the liquid phase, $v_{a}^{*}$ is a constant given by $v_{a}^{*}=\left(3 \sqrt{4 \pi} v_{a}\right)^{2 / 3}$ where $v_{a}$ is the molecular volume of the liquid and $\tilde{\sigma}$ is the interfacial free energy per unit area appearing because an interface between the solid nucleus and surrounding liquid is formed. In terms of the number of particles of the cluster with critical size $n_{\text {crit }}$, the free energy difference may be written in the form

$$
\Delta G(n)=\Delta G_{\text {crit }}\left[3\left(\frac{n}{n_{\text {crit }}}\right)^{2 / 3}-2\left(\frac{n}{n_{\text {crit }}}\right)\right],
$$

where the height of the barrier is given by $\Delta G_{c r i t}=\frac{16 \pi}{3} v_{a}^{2} \tilde{\sigma}^{3} / \Delta \mu^{2}$.

Using Eq. (47) and the rules of mesoscopic nonequilibrium thermodynamics, the resulting Fokker-Planck equation for the probability distribution is

$$
\begin{aligned}
& \frac{\partial f}{\partial t}+\nabla \cdot(\mathbf{u} f)=\frac{\partial}{\partial n}\left(\frac{D_{n}}{k_{B} T} f \frac{\partial}{\partial n}\left[\Delta G(n)+\frac{m(n)}{2}\left(\mathbf{u}-\mathbf{v}_{0}\right)^{2}\right]+D_{n} \frac{\partial f}{\partial n}\right) \\
& +\frac{\partial}{\partial \mathbf{u}} \cdot\left\{\underline{\beta} \cdot\left(\mathbf{u}-\mathbf{v}_{0}\right) f-\underline{\tau} \cdot \mathbf{F} f+\mathbb{D}_{v} \cdot \frac{\partial f}{\partial \mathbf{u}}\right\} .
\end{aligned}
$$

Here $D_{n}$ plays the role of a diffusion coefficient in cluster size space. Reducing the description to the diffusion regime $\left(t \gg \beta^{-1}\right)$, an effective value for the nucleation barrier was obtained: 
$\Delta \tilde{G}=\Delta G+(3 / 2) k_{B} T \ln |n|$. This result is interesting because it implies that the flow modifies the nucleation rate in the form

$$
J \simeq k^{+}\left(n_{\text {crit }}\right) e^{\Delta \tilde{G}_{\text {crit }} / k_{B} T},
$$

where $k^{+}$is the rate of attachment of particles to the cluster and $\Delta \tilde{G}_{c r i t}$ is the height of the nucleation barrier.

\subsection{Extension of the classical nucleation theory}

A different approach to the problem of nucleation in the presence of flow was proposed in Ref. (Blaak, 2004) by extending the classical nucleation theory in an intuitive and interesting form. Starting from Eq. (49), a macroscopic nonequilibrium equation of state was proposed for the Gibbs free energy difference by expanding in powers of the shear rate about the equilibrium values of both, the chemical potential difference and the surface energy

$$
\begin{array}{r}
\Delta \mu=\Delta \mu^{e q}\left[1+c_{0} \dot{\gamma}^{2}+O\left(\dot{\gamma}^{4}\right)\right], \\
\tilde{\sigma}=\tilde{\sigma}^{e q}\left[1+\kappa_{0} \dot{\gamma}^{2}+O\left(\dot{\gamma}^{4}\right)\right],
\end{array}
$$

where $c_{0}$ and $\kappa_{0}$ are, in principle, fitting constants and $\Delta \mu^{e q}$ and $\tilde{\sigma}^{e q}$ are the equilibrium values of the chemical potential difference and the surface energy. The second term at the right hand side of Eq. (52) is similar to the corrections previously obtained from MNET, see Eq. (21). The main difference is that in the latter case these corrections enter through the local kinetic energy of the system. Using these dependences, it was shown that the barrier height and the critical cluster size $n_{\text {crit }}$ depend on the shear in the form

$$
\begin{aligned}
\Delta G_{c r i t} & =\frac{16 \pi v_{s}\left[\tilde{\sigma}^{e q}\right]^{3}}{3\left[\Delta \mu^{e q}\right]^{2}}\left[1+\left(3 \kappa_{0}-2 c_{0}\right) \dot{\gamma}^{2}+O\left(\dot{\gamma}^{4}\right)\right], \\
n_{\text {crit }} & =\frac{32 \pi v_{s}\left[\tilde{\sigma}^{e q}\right]^{3}}{3\left[\Delta \mu^{e q}\right]^{3}}\left[1+\left(3 \kappa_{0}-3 c_{0}\right) \dot{\gamma}^{2}+O\left(\dot{\gamma}^{4}\right)\right] .
\end{aligned}
$$

Brownian dynamic simulations performed with relatively small shear rates showed a shift of the maximum of the free energy difference as a function of the applied shear rate implying an increase in the nucleation barrier and the critical nucleation size (Blaak, 2004).

The authors conlcuded that their results indicate that crystal nucleation is suppressed by the presence of shear (Butler \& Harrowell, 1995), however much larger shear rates were not accessed by simulations because the increase of the critical nucleus size would lead to simulation artefacts. However, this study seems to be necessary because experimental observations on lyzosyme, ferritin and apoferritin crystallization reported in Ref. (A. Penkova, 2006), showed that the presence of moderate flows may promote nucleation whereas large flows may suppress the nucleation. As a consequence, there is still an open question on the appropriate theoretical description of the effect of flow on nucleation.

\subsection{Flow induced nucleation: A Control volume thermodynamics approach}

Here, we will formulate a novel model of flow induced nucleation by using control volume thermodynamics. The general idea is very simple and goes through the lines indicated along this chapter, that is, by assuming that nonequilibrium equations of state can be formulated in a way consistent with the laws of thermodynamics when a flow is imposed on the system. 
Consider that the system is an open control volume with size $V$ that allows the transit of particles in solution through it and that, on average, remains with stationary pressure and temperature. The first law of thermodynamics for open systems states that the change of the total energy $E$ of the system obeys the relation

$$
\Delta E=Q+W
$$

where $Q$ and $W$ are the amount of heat and work exchanged between the system and the surroundings, and the change in the total energy is given by the relation

$$
\Delta E=\Delta U+\Delta E_{c}+\Delta E_{p}
$$

Here, $U$ is the internal energy of the system and $E_{c}$ and $E_{p}$ are their kinetic and potential energies. Assuming that the potential energy contribution is negligible in the case of our interest, then $E_{c}$ is the kinetic energy of the particles in solution and we may write

$$
E=U+\frac{1}{2} m N \bar{v}^{2}+\frac{1}{2} \rho V \bar{v}^{2},
$$

where $m$ is the mass of a particle, $N$ is the total number of particles in the control volume and $\rho$ is the density of the solvent, that we assume constant. Thus, the total mass of the solution $m N$ crosses the container volume with average velocity $\bar{v}$.

In equilibrium, the Gibbs free energy $G$ is defined in terms of the internal energy $U$, the entropy $S$ and the total volume of the system $V$ :

$$
G(N, P, T)=U-T S+P V,
$$

where $P$ is the pressure. However, in order to be consistent with the first principle (55) for an open system, in formulating the definition of the free energy we have to take into account not only the internal energy $U$, but the total energy $E$ of the system. Otherwise, it may occur that the free energy difference could not be equal to the work exchanged between the system and the surroundings. In view of these considerations, we propose the nonequilibrium free energy

$$
G^{n e q}(N, P, T, \bar{v})=E-T S+P V .
$$

Now, by using Euler's thermodynamic equation, $U=T S-P V+\mu N$, one can write the nonequilibrium Gibbs free energy in terms of the chemical potential $\mu$ and the kinetic energy of particles in solution in the form

$$
G^{n e q}=\mu N+\frac{1}{2} m N \bar{v}^{2}+\frac{1}{2} \rho V \bar{v}^{2} .
$$

Using Eq. (58), we may define the effective chemical potential of the particles in solution $\mu^{\text {neq }}$

$$
\mu^{n e q}=\mu+\frac{1}{2} m \bar{v}^{2} .
$$

Notice that Eq. (59) is fully compatible with Eq. (52) and with (21). However, it is important to stress that Eq. (59) is exact and a natural consequence of the first law of thermodynamics for open systems. 


\subsection{The kinetic energy contribution to nucleation}

Consider now that nucleation of crystals/aggregates may occur in the system. Following the lines of classical nucleation theory, the description of the nucleation process can be given by means of the free energy difference between the solution $G_{S}^{\text {neq }}$ and the solution/cluster compound $G_{c}^{n e q}$. Thus, assuming that the particles in solution move at a constant average velocity $\bar{v}$, that is, local diffusion is negligible, the Gibbs free energy of the particles in solution $G_{S}^{n e q}$ takes the form

$$
G_{s}^{n e q}=\mu_{s}^{n e q} n_{s}+\frac{1}{2} \rho V \bar{v}^{2},
$$

where $\mu_{s}$ and $n_{s}$ are the chemical potential and the number of particles in solution, respectively. When the cluster of particles starts to form, the Gibbs free energy of the system changes and therefore, the solution/cluster compound $G_{C}$ becomes

$$
G_{c}^{n e q}=\left(n_{s}-n_{c}\right) \mu_{s}^{n e q}+\mu_{c} n_{c}+\tilde{\sigma} A+\frac{1}{2} \rho V \bar{v}^{2},
$$

where $\mu_{c}$ and $n_{c}$ are the chemical potential and the number of particles of the cluster with surface energy $\tilde{\sigma}$ and surface $A$. Using Eqs. (60) and (61), one obtains the following expression for the total change in the Gibbs free energy of the system $\Delta G^{\text {neq }}=G_{c}^{\text {neq }}-G_{s}^{\text {neq }}$

$$
\Delta G^{n e q}=-\Delta \mu^{n e q} \frac{4 \pi R^{3}}{3 v_{s}}+4 \pi \gamma R^{2}
$$

where $\Delta \mu^{n e q}=\mu_{s}-\mu_{c}+\frac{1}{2} m \bar{v}^{2}=\Delta \mu+\frac{1}{2} m \bar{v}^{2}$ and we have assumed that the cluster has spherical shape with radius $R$. Eq. (62) shows that for a constant value of $\Delta \mu$, the Gibbs free energy free energy barrier decreases for increasing values of the flow velocity $\bar{v}$. This means that the flow gives energy to the particles in order to overcome the barrier. This result agrees partially with those reported in (Blaak, 2004) and, more importantly, with the experimental observation (A. Penkova, 2006) that for low and moderate flow velocities nucleation is enhanced. However, it disagrees with the experimental fact that for large flow velocities nucleation is inhibited or supressed. To explain this second effect, it is necessary to consider the deformation of the nuclei. For another discussion on nucleation in soft-matter colloid-type systems, see (Gadomski, 2005; 2006).

\subsection{The elastic contribution}

The stresses applied by the solvent on the nucleus' surface will induce its deformation and, as a consequence, an elastic contribution to the free energy $G_{e l}$ will emerge. This contribution is proportional to the restitution force that tends to maintain the spherical shape of the nucleus of volume $V_{c}$ and, consequently, it is proportional to the elastic stress per unit volume $P^{E}=$ $\operatorname{tr}\left[\mathbb{P}^{E}\right]$, thus leading to the expression: $G_{e l} \propto P^{E} V_{c}$.

The average elastic stress per unit volume of the solution/cluster compound is given through the relation: $\mathbb{P}^{E}=-\xi_{0} F_{0} \mathbb{A}$ with $\mathbb{A}$ the conformation tensor accounting for the average deformation of the nuclei. In the present case, the characteristic restitution coefficient per unit mass $\xi_{0}$ will be related to the Lame coefficients of the material and the dimensionless factor $F_{0}$ will be held constant for simplicity. Thus, from Eq. (37), the average deformation of the nuclei in terms of the flow velocity is

$$
\mathbb{A}=\frac{k T}{F_{0}} \mathbb{I} \cdot\left(\mathbb{I}-\frac{L_{R r}}{L_{R R} F_{0}}\left(\nabla \mathbf{v} \cdot \nabla \mathbf{v}^{T}\right)\right)^{-1},
$$




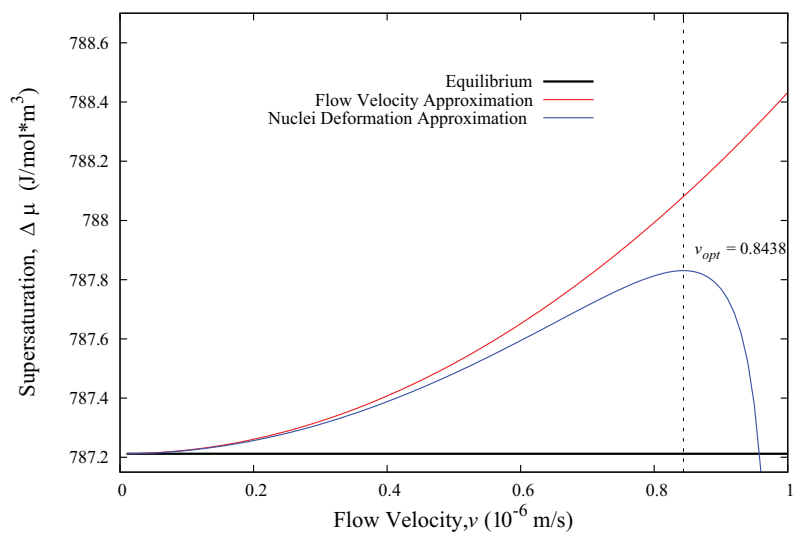

Fig. 4. Supersaturation as a function of the averge flow velocity for a lysozime solution as given by Eq. (67). The existence of an optimal flow velocity $\bar{v}_{\text {opt }}$ gives rise to two nulceation regimes. For moderate flow velocities $\bar{v}<\bar{v}_{\text {opt }}$ nucleation is enhanced whereas for large flow velocities $\bar{v}>\bar{v}_{\text {opt }}$ becomes inhibited and even supressed.

where we have assumed stationary conditions: $d \mathbb{A} / d t-2[\mathbb{A} \cdot \nabla \mathbf{v}]^{s}=0$ and used the relation $\langle\vec{F} \vec{R}\rangle=F_{0} \mathbf{A}$. Now, assuming that the average flow is in the $x$-direction and calculating the trace of $\mathbb{A}$ we may finally write

$$
P^{E} \approx-\xi_{0} k T\left(\frac{\tilde{a}}{\tilde{a}-b \bar{v}^{2}}\right)
$$

where $\tilde{a}=L_{R R} F_{0}$ and $b=L_{R r} R_{c r i t}^{-2}$. Here we have assumed that $|\nabla \mathbf{v}| \sim \bar{v} / R_{\text {crit }}$ with the characteristic length given by the radius of the critical nucleus $R_{\text {crit }}$. Eq. (64) shows that the elastic contribution to the stresses in the nuclei is a function of the temperature $T$ and the average flow velocity $\bar{v}$. This effect modifies the total free energy of the particles in the solution/cluster compound $G_{c}$ by adding the term $G_{e l}=\Delta P^{E} V_{c}$, with $\Delta P^{E}=P^{E}-P_{0}^{E}$ with $P_{0}^{E}=-\xi_{0} k T$ the value of $P^{E}$ at zero flow. Therefore, the expression for the Gibbs free energy taking into account the elastic contribution is

$$
G_{c}=\left(n_{s}-n_{c}\right) \mu_{s}^{n e q}+\mu_{c} n_{c}+\gamma A+\Delta P^{E} V_{c} .
$$

Now, using Eqs. (60) and (65), the total change in the Gibbs free energy in terms of the radius of the nucleus is given by

$$
\Delta G^{n e q}=-\Delta \tilde{\mu}^{n e q} \frac{4 \pi R^{3}}{3 v}+4 \pi \gamma R^{2}
$$

where

$$
\Delta \tilde{\mu}^{n e q}=\Delta \mu+\frac{1}{2} m \bar{v}^{2}+\xi_{0} v_{s} k T\left(1-\frac{\tilde{a}}{\tilde{a}-b \bar{v}^{2}}\right) .
$$

This result implies that the steady state nucleation rate $J$ becomes a function of the average flow velocity

$$
J(\bar{v}) \simeq \exp \left[-\Delta G_{c r i t}^{n e q}(\bar{v}) / k T\right]
$$


because the height of the free energy barrier becomes a function of $\bar{v}: \Delta G_{c r i t}^{n e q} \sim \tilde{\sigma}^{3} /\left[\Delta \tilde{\mu}^{n e q}\right]^{2}$, see Eq. (53). The main consequence arising from the last term of (67) is that an optimal average flow velocity $\bar{v}_{\text {opt }}$ appears. This means that there are two regimes for nucleation in the presence of flow. For moderate flow velocities, $\bar{v}<\bar{v}_{o p t}$, nucleation is enhanced whereas for large flow velocities $\bar{v}>\bar{v}_{\text {opt }}$ nucleation is inhibited or even supressed. The existence of an optimal flow velocity for nucleation was established experimentally in Ref. (A. Penkova, 2006) for lyzosyme, ferritin and apoferritin solutions.

\section{Conclusions}

In this chapter, we have discussed three important aspects of thermodynamics in the presence of flow. By considering different points of view ranging from kinetic and stochastic theories to thermodynamic theories at mesoscopic and macroscopic levels, we addressed the effects of flow on transport coefficients like diffusion and viscosity, constitutive relations and equations of state. In particular, we focused on how some of these effects may be derived from the framework of mesoscopic nonequilibrium thermodynamics.

Our analysis started by considering how the flow introduces corrections on transport coefficients and illustrated how this may be relevant for giving a sound theoretical description of experiments. The first case considered was the contribution on the diffusion coefficient due to a shear flow. This contribution, proportional to the velocity gradient or shear rate, was first obtained by means of kinetic theory for Lorentz fluids and after that corroborated in a more general fashion by MNET and Langevin calculations. Computer simulations and experiments in which this correction was observed were also discussed. Comparison with experiments illustrating how these contributions are relevant for giving a sound theoretical explanation of the shear induced effect and the transition to the irreversibility was also presented.

Afterwards, we studied the existence of non-linear constitutive relations on several systems like, for example, suspensions of Brownian and non-Brownian particles and polymer solutions. These non-linear relations are understood in the sense that the currents, like for instance, the diffusion current or the momentum current are not simply proportional to their conjugated thermodynamic forces. In particular, the relation between the stress tensor of a fluid and the velocity gradient, which is linear for simple Newtonian fluids, becomes non-linear for many soft-matter systems like polymer solutions, polymer melts, glasses, etc. This fact makes these materials non-Newtonian and viscoelastic. We illustrated the phenomenological approach to this problem by mentioning Maxwell and extended Maxwell fluid models, and then showed how similar non-linear constitutive relations emerge from the mesoscopic thermodynamics analysis by considering Faxén's theorem and its generalizations to the case of time dependent flows.

These analyses allowed us to present a discussion on how the equilibrium equations of state become modified when the system is subject to sufficiently strong flows, because not only the non-diagonal components of the stress tensor become modified, but also the normal components. In addition, through the discussion we have shown that the chemical potential becomes corrected by a term proportional to the local kinetic energy of diffusion, and therefore, is a flow dependent quantity. This correction was analyzed in the context of nucleation, were it was found that both the nucleation barrier and consequently the nucleation rate can be written in terms of the kinetic energy of the flow.

Using control volume thermodynamics, we showed that the nonequilibrium equations of state heuristically proposed by Frenkel and co-workers is in fact consistent with the general expression of the first law of thermodynamics, in which the heat and the work exchanged 
between the system and its surroundings are responsible for the change of the internal, kinetic and potential energies of the system. Our proof uses arguments similar to those previously used by Oono's when discussing why nonequilibrium thermodynamics may treat both thermodynamic and hydrodynamic fluctuations. Finally, we have proposed a model taking into account internal restitutive stresses of the nucleus that allows to describe in a qualitative form the experiments on lysozyme, ferritin and apoferritin nucleation in the presence of flow. The main result is that the supersaturation parameter and consequently the nucleation rate exhibit two regimes. One in which nucleation is enhanced by low and moderate flow velocities and other one (large flow velocities) in which nucleation is inhibited or even supressed by the flow. These results lead to the existence of an optimal nucleation flow velocity, a quantity that was reported in experiments.

\section{Acknowledgments}

We thank Dr. G. Barrios for kindly sharing Fig. 1 with us. This work was supported by Grant DGAPA-UNAM IN-102609. Rl and RLF also acknowledge financial support by CONACyT.

\section{References}

A. Penkova, e. a. (2006). Nucleation of protein crystals under the influence of solution shear flow., Annals of the New York Academy of Sciences 1077: 214-31.

Abou, B. \& Gallet, F. (2004). Probing a nonequilibrium einstein relation in an aging colloidal glass, Phys. Rev. Lett. 93: 160603.

Aidun, C. K. \& Ding, E. J. (1998). Direct analysis of particulate suspensions with inertia using the discrete Boltzmann equation, J. Fluid Mech. 373: 287.

Apelian, M. R., e. a. (1988). Impact of the constitutive equation and singularity on the calculation of stick-slip flow: The modified upper-convected maxwell model, $J$. Non-Newtonian Fluid Mech. 27: 299-321.

Beck, C. \& Cohen, E. (2003). Superstatistics, Physica A 322: 267-275.

Bellon, L. e. a. (2001). Violation of the fluctuation-dissipation relation during the formation of a colloidal glass, Europhys. Lett. 53: 511.

Beris, A. N. \& Edwards, B. J. (1994). Thermodynamics of flowing systems, Oxford, New York.

Blaak, R., e. a. (2004). Homogeneous nucleation of colloidal melts under the influence of shearing fields, J. of Phys.: Cond. Matt. 16: S3873-S3884.

Bossis, G. \& Brady, J. F. (1989). The rheology of brownian suspensions, J. Chem. Phys 91: 1866.

Breedveld, V., e. a. (1998). The measurement of the shear-induced particle and fluid tracer diffusivities in concentrated suspensions by a novel method, J. Fluid Mech. 375: 297-318.

Butler, S. \& Harrowell, P. (1995). Kinetics of crystallization in a shearing colloidal suspension, Phys. Rev. E 52: 6424-6430.

C. Beck, E. C. \& Swinney, H. (2005). Fluctuations of entropy and log-normal superstatistics, Phys. Rev. E 72: 056133.

Callen, H. B. (1985). Thermodynamics and an introduction to thermostatistics, John Wiley and Sons, New York, Second Edition.

Criado-Sancho, M. e. a. (2005). Nonequilibrium kinetic temperatures in flowing gases, Phys. Lett. A 350: 339.

de Groot, S. R. \& Mazur, P. (1984). Non-equilibrium Thermodynamics, Dover, New York.

Delgado, J. \& Castillo, R. (2005). Unpublished data . 
Delgado, J. \& Castillo, R. (2007). Shear-induced structures formed during thixotropic loops in dilute worm-micelle solutions, J. Coll. Int. Sci. 312: 481.

Demirel, Y. (2007). Non-equilibrium Thermodynamics, Elsevier, Amsterdam.

Doi, M. \& Edwards, S. F. (1998). The Theory of Polymer Dynamics, Oxford University Press, New York.

Drazer, D., e. a. (2002). Deterministic and stochastic behaviour of non-brownian spheres in sheared suspensions, J. Fluid Mech. 460: 307-335.

Drossinos, Y. \& Reeks, M. W. (2005). Brownian motion of finite-inertia particles in a simple shear flow, Phys. Rev. E 71: 031113.

Ferziger, J. H. \& Kaper, H. G. (1972). Mathematical Theory of Non-Uniform Gases, North Holland, Amsterdam.

Gadomski, A. e. a. (2005). On temperature and space-dimension dependent matter agglomerations in a mature growing stage, Chemical Physics 310: 153-161.

Gadomski, A. e. a. (2006). Formation of soft material in terms of a coupled matter migration-and-relaxation picture: a synchronous mode, Materials Science-Poland 24(No. 4): 935-949.

Gadomski, A. e. a. (2008). Statistical Mechanics Research, Nova Science Publishers, Inc. New York, chap. 1, pp. 13-98.

Guasto, J. S. \& Gollub, J. P. (2007). Hydrodynamic irreversibility in particle suspensions with nonuniform strain, Phys. Rev. E 81: 061401.

Hinch, E. J. (1994). Uncoiling a polymer molecule in a strong extensional flow, J. Non-Newtonian Fluid Mech. 54: 209-230.

James, D. F. (1972). Constitutive Equations for Dilute Polymer Solutions, J. Rheol. 16: 175.

Kondepudi, D. K. \& Prigogine, I. (1999). Modern Thermodynamics: From Heat Engines to Dissipative Structures, John Wiley and Sons, Chichester.

Kurchan, J. (2005). In and out of equilibrium, Nature 433: 222-225.

Ladd, A. J. C. (1994). Numerical simulations of particulate suspensions via a discretized Boltzmann equation. Part 1 and Part 2., J. Fluid Mech. 271: 285-339.

Málaga, C., e. a. (2006). Mesoscopic constitutive relations for dilute polymer solutions, Physica A 369: 291-300.

Marchetti, M. C. \& Dufty, J. W. (1983). Tagged particle fluctuations in uniform shear flow, J. Stat. Phys. 32: 255-277.

Mauri, R. \& Leporini, D. (2006). Violation of the fluctuation-dissipation theorem in confined driven colloids, Europhys. Lett. 76: 10221028.

Mazur, P. \& Bedeaux, D. (1974). A generalization of faxén's theorem to nonsteady motion of a sphere through an incompressible fluid in arbitrary flow, Physica A 76: 235-246.

McLennan, J. A. (1989). Introduction to Non-equilibrium Statistical Mechanics, Prentice-Hall, Englewood Cliffs, New Jersey.

Meyers, M. A. \& Chawla, K. K. (2009). Mechanical Behavior of Materials, Cambridge University Press, Cambridge.

O'Hern, C. S. e. a. (2004). Effective temperatures in driven systems: Static versus time-dependent relations, Phys. Rev. Lett. 93: 165702.

Onuki, A. (2004). Phase Transition Dynamics, Cambridge University Press, New York.

Pérez-Madrid, A. (2005). A model for nonexponential relaxation and aging in dissipative systems, J. Chem. Phys. 122: 214914.

Pérez-Madrid, A. \& Santamaría Holek, I. (2009). Fluctuation theorems for systems under Fokker-Planck dynamics, Phys. Rev. E 79: 011101. 
Pérez-Madrid, e. a. (1994). Brownian motion in the presence of a temperature gradient, Physica A 212: 231-238.

Pine, D. J., e. a. (2005). Chaos and threshold for irreversibility in sheared suspensions, Nature 438: 997-1000.

Popov, A. V. \& R., H. (2007). Ontology of temperature in nonequilibrium systems, J. Chem. Phys. 126: 244506.

Ragone, D. V. (1995). Thermodynamics of materials, John Wiley and Sons, New York.

Rallison, J. M. \& Hinch, E. J. (1988). Do we understand the physics in the constitutive equation?, J. Non-Newtonian Fluid Mech. 29: 37-55.

Reguera, D. \& Rubi, J. (2003a). Homogeneous nucleation in inhomogeneous media. i. nucleation in a temperature gradient, J. Chem. Phys. 119: 9877.

Reguera, D. \& Rubi, J. (2003b). Homogeneous nucleation in inhomogeneous media. ii. nucleation in a shear flow, J. Chem. Phys. 119: 9888.

Rodríguez, R. F., e. a. (1983). Fokker-Planck and Langevin descriptions of fluctuations in uniform shear flow, J. Stat. Phys. 32: 279-298.

Rodríguez, R. F. \& Santamaría Holek, I. (2007). Superstatistics of brownian motion: A comparative study, Physica A 385: 456-464.

Rubi, J. M. \& Mazur, P. (1994). Simultaneous brownian motion of n particles in a temperature gradient, Physica A 250: 253-264.

Rubi, J. M. \& Pérez-Madrid, A. (1999). Inertial effects in non-equilibrium thermodynamics, Physica A 264: 492.

Ryskin, G. (1988). Brownian motion in a rotating fluid: Diffusivity is a function of the rotation rate Phys. Rev. Lett. 61: 01442.

Santamaría Holek, I., e. a. (2005). Mesoscopic thermodynamics of stationary non-equilibrium states, New J. Phys. 7: 35.

Santamaría Holek, I., e. a. (2009). Transition to irreversibility in sheared suspensions: An analysis based on a mesoscopic entropy production, Phys. Rev. E 79: 031201.

Santamaría Holek, I., e. a. (2001). Diffusion in stationary flow from mesoscopic nonequilibrium thermodynamics, Phy. Rev. E 63: 051106.

Sarman, S., e. a. (1992). Mutual and self-diffusion in fluids undergoing strong shear, Phys. Rev A 46: 893-902.

Seriou, A. \& Brady, J. F. (2004). Shear-induced self-diffusion in non-colloidal suspensions, J. Fluid Mech. 506: 285-314.

Shea, J. E. \& Oppenheim, I. (1998). Fokker-Planck and non-linear hydrodynamic equations of an inelastic system of several brownian particles in a non-equilibrium bath, Physica A 250: 265.

Subramanian, G. \& Brady, J. F. (2004). Multiple scales analysis of the Fokker-Planck equation for simple shear flow, Physica A 334: 343-384.

Swailes, D. C., e. a. (2009). Stochastic transport of particles in straining flows, Phys. Rev. E 79: 036305.

Taylor, G. I. \& Friedman, J. (1996). Low Reynolds Number Flows, National Committe on Fluid Mechanics Films, Encyclopedia Britannica Educational Corp., United States. 


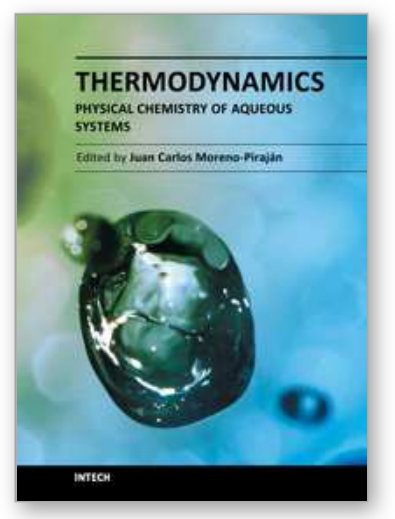

\author{
Thermodynamics - Physical Chemistry of Aqueous Systems \\ Edited by Dr. Juan Carlos Moreno Pirajễin
}

ISBN 978-953-307-979-0

Hard cover, 434 pages

Publisher InTech

Published online 15, September, 2011

Published in print edition September, 2011

Thermodynamics is one of the most exciting branches of physical chemistry which has greatly contributed to the modern science. Being concentrated on a wide range of applications of thermodynamics, this book gathers a series of contributions by the finest scientists in the world, gathered in an orderly manner. It can be used in post-graduate courses for students and as a reference book, as it is written in a language pleasing to the reader. It can also serve as a reference material for researchers to whom the thermodynamics is one of the area of interest.

\title{
How to reference
}

In order to correctly reference this scholarly work, feel free to copy and paste the following:

I. Santamaría-Holek, R. Lugo-Frías, R. F. Rodríguez and A. Gadomski (2011). Mesoscopic Thermodynamics in the Presence of Flow, Thermodynamics - Physical Chemistry of Aqueous Systems, Dr. Juan Carlos Moreno PirajÃ $i n(E d),. ~ I S B N:$ 978-953-307-979-0, InTech, Available from:

http://www.intechopen.com/books/thermodynamics-physical-chemistry-of-aqueous-systems/mesoscopicthermodynamics-in-the-presence-of-flow

\section{INTECH}

open science | open minds

\section{InTech Europe}

University Campus STeP Ri

Slavka Krautzeka 83/A

51000 Rijeka, Croatia

Phone: +385 (51) 770447

Fax: +385 (51) 686166

www.intechopen.com

\section{InTech China}

Unit 405, Office Block, Hotel Equatorial Shanghai

No.65, Yan An Road (West), Shanghai, 200040, China

中国上海市延安西路65号上海国际贵都大饭店办公楼 405 单元

Phone: +86-21-62489820

Fax: $+86-21-62489821$ 
(C) 2011 The Author(s). Licensee IntechOpen. This chapter is distributed under the terms of the Creative Commons Attribution-NonCommercialShareAlike-3.0 License, which permits use, distribution and reproduction for non-commercial purposes, provided the original is properly cited and derivative works building on this content are distributed under the same license. 